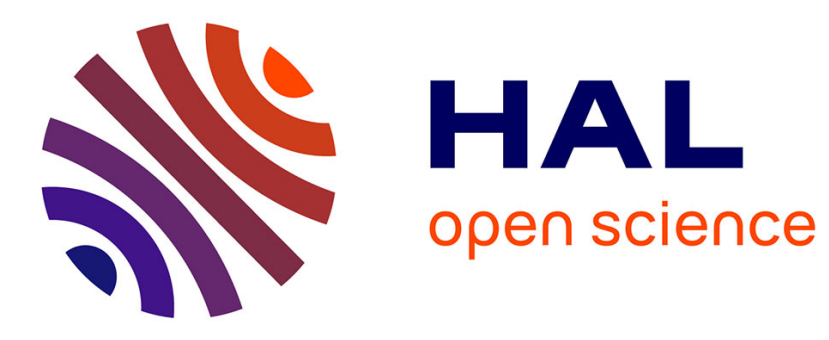

\title{
Wireless Field Buses for Aerospace Ground and In-Flight Testing: an Experiment
}

Julien Henaut, Akram Hakiri, Pascal Berthou, Daniela Dragomirescu, Thierry Gayraud, Robert Plana

\section{- To cite this version:}

Julien Henaut, Akram Hakiri, Pascal Berthou, Daniela Dragomirescu, Thierry Gayraud, et al.. Wireless Field Buses for Aerospace Ground and In-Flight Testing: an Experiment. FET 2009 - Fieldbuses and nEtworks in Industrial \& Embedded Systems, May 2009, South Korea. pp.1. hal-00436716

\section{HAL Id: hal-00436716 https://hal.science/hal-00436716}

Submitted on 27 Nov 2009

HAL is a multi-disciplinary open access archive for the deposit and dissemination of scientific research documents, whether they are published or not. The documents may come from teaching and research institutions in France or abroad, or from public or private research centers.
L'archive ouverte pluridisciplinaire HAL, est destinée au dépôt et à la diffusion de documents scientifiques de niveau recherche, publiés ou non, émanant des établissements d'enseignement et de recherche français ou étrangers, des laboratoires publics ou privés. 


\title{
Wireless Field Buses for Aerospace Ground and In-Flight Testing: an Experiment
}

\author{
Julien HENAUT*,** Akram HKIRI*,** Pascal BERTHOU*,** \\ Daniela DRAGOMIRESCU*,** Thierry GAYRAUD*,** Robert PLANA*,** \\ *CNRS; LAAS; 7, avenue du Colonel Roche, F-31077 Toulouse, France(jhenaut@laas.fr, daniela@laas.fr) \\ ** Université de Toulouse; UPS, INSA, INP, ISAE; LAAS; F-31077 Toulouse, France
}

\begin{abstract}
Ultra-Wideband (UWB) radio has been proposed for physical layer standard for high Speed wireless personal area network (WPANs). One proposal is referred to as Multiband Orthogonal Frequency Division Multiplexing (MB-OFDM). This paper proposes to evaluate this powerful modulation scheme in a wireless field bus for aerospace industry hardware system testing. Simulation results show the feasibility and the limits of a software simulation approach. Measurements were done indoor, on Airbus wings and inside à mock-up satellite to determine the real propagation channel, in order to reproduce theses environments in the lab.

Frame error rate measurements show a good accuracy of the simulation model for the indoor environment. For the outdoor environment, a lot of measurements were done. Some of these results are presented in this paper. Their strongly varying behavior has to be investigated further.
\end{abstract}

Keywords: wireless field bus, aerospace applications, UWB, MB-OFDM measurements, WiMEDIA

\section{INTRODUCTION}

To evaluate a system's compliance with its specified requirements, Hardware System Testing is conducted on the complete and integrated system. This phase is essential in all industry branches, especially in the very regulated and critical aerospace world. In the final phase of the development of an airplane, flight test equipment gathers and analyzes data during flight to evaluate the flight characteristics of the aircraft and validate its design, including safety aspects. One of the most critical tests is the measurement of the pressure around the wings during flight. All new aircrafts are computer designed with the use of virtual wind tunnels. So, very accurate measurements have to be done on the aircraft to validate the model before the aircraft can be industrially produced. In the case of satellites, vibration and mechanical stress are two critical phenomena a satellite endures during launch. This is leading to the necessity for accurate ground tests using strain gauges or thermal sensors before allowing a launch. All such systems used by aircraft and satellite manufacturers today are wired systems. Sensors put around the wings or inside the satellite compartments are wired to a concentrator inside the cabin or the operator's room. Although good performance is observed in terms of measurement accuracy, these systems have strong drawbacks. The two most important ones are the weight of the system and its cost as well as the cost of its installation.

These points and the complexity of such systems do not allow a great number of measurement points. This is why research is done on a wireless measurement system based on wireless sensors networks (WSNs). Detailed specifications of the two targeted use cases can be found in (Henaut et al. 2009).

This paper is organized into seven sections: After a brief introduction, section II introduces the reasons for the choice of an Ultra Wideband (UWB) modulation. The development of a Wimedia protocol stack, associated simulation results and limitations are presented in section III. The methodology that allows reproduction of measurements in the lab is presented in section IV, the measurement protocol in section $\mathrm{V}$ and the Bit Error Rate measurements for the different channels in section VI. Finally conclusions are drawn in the last section.

\section{CHOICE OF A MODULATION}

As described in Henaut et al. (2008), the radio interface remains one of the bottlenecks to implement high data rate truly low-power WSNs. Simple binary modulations as PSK (Phase Shift Keying) are often considered, but Henaut et al. (2008) shows that the radiated power must be high. Thus, such a network will interfere with other systems and consequently not be allowed by aerospace regulation authorities. In order to reduce interferences, an Ultra Wideband modulation has been chosen. The regulations allow radio transmission in the frequency range from 3.1 $\mathrm{GHz}$ to $10.6 \mathrm{GHz}$ (6 GHz to $8.5 \mathrm{GHz}$ in Europe), at a limited transmit power of $-41.3 \mathrm{dBm} / \mathrm{MHz}$. In Europe, such a system is very coexistence friendly because there are no aerospace critical systems in this frequency range (like Microwave Landing Systems at 5.02-5.1 GHz). Although Impulse Radio has strong potential (Lecointre et al. 2008), the leading technology for use in high speed wireless UWB communication is the Multi Band-Orthogonal Frequency Division Multiplexing (MB-OFDM). This technique was first developed by IEEE $802.15 .3 \mathrm{a}$ and standardized by ECMA (ECMA 2008) to be very similar to many conventional wireless OFDM systems. The spectrum is divided in 14 subbands, each having a bandwidth of $528 \mathrm{MHz}$. The main advantages are described in Batra et al. (2004). One of the most important is the capacity of capturing energy in dense multipath environments with a simple implementation. This advantage is one of the decisive points in the choice of MBOFDM as the Wimedia UWB Physical Layer (PHY). 


\section{EVALUATION WITH SIMULATIONS}

Prior to any circuit development, simulations were used to validate the possibility of using a Wimedia compliant architecture.

The simulation was made using NS-2. This simulator does not provide a Wimedia-UWB Layer, so we developed the protocol stack presented in figure 1. The UWB Physical Layer manages the transmission of bits over the antenna. The Wimedia Mac Layer provides all means and services for frame transmission between the physical layer and all other higher layers. Beacons, Distributed Reservation Protocol (DRP) and Prioritized Contention Access (PCA) Layers can be chosen regarding the need of the protocol: the Beacon module is responsible for beaconing protocol; DRP and PCA manage the super frame reservation using both dynamic reservation and contention protocol. High level layers are designed to support high rate communication and higher throughput.

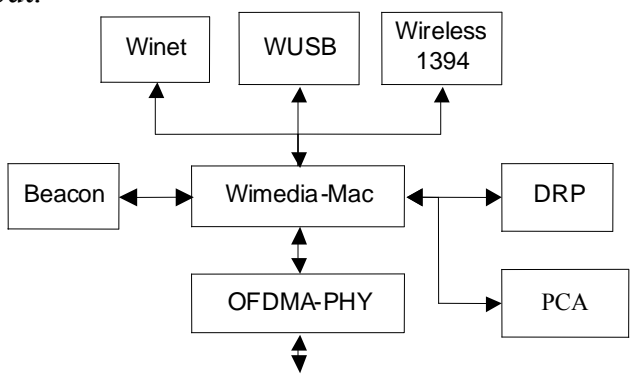

Fig. 1. Wimedia Stack Protocol

The class diagram points out the Physical Layer of the Wimedia-UWB protocol. The main class is the WimediaPhy which is used to manage all communication through the antenna. The techniques of Modulation implemented within the UWB communication module are both QPSK and DCM. A New physical state was implemented within the Physical Layer called OFDM_PHY_STANDBY to model an advanced antenna state in order to support the management of the node hibernation.

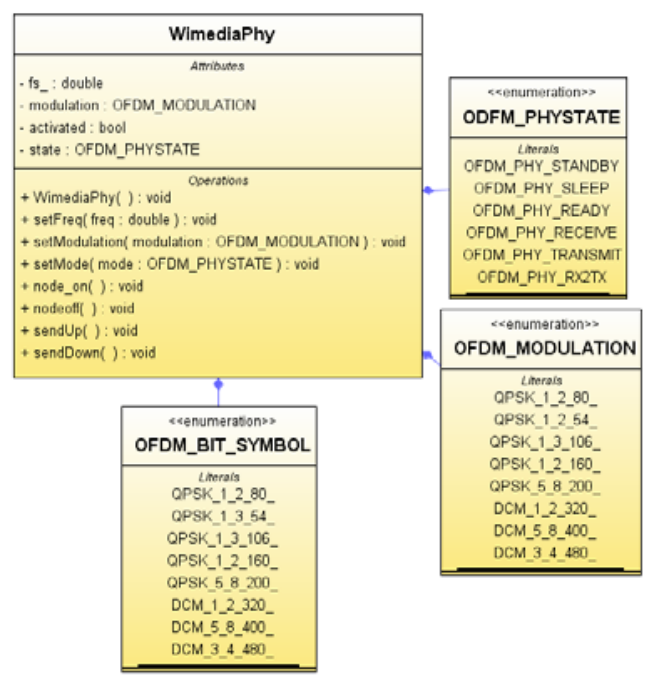

Fig. 2. Wimedia Physical Layer (MB-OFDM) Class Diagram

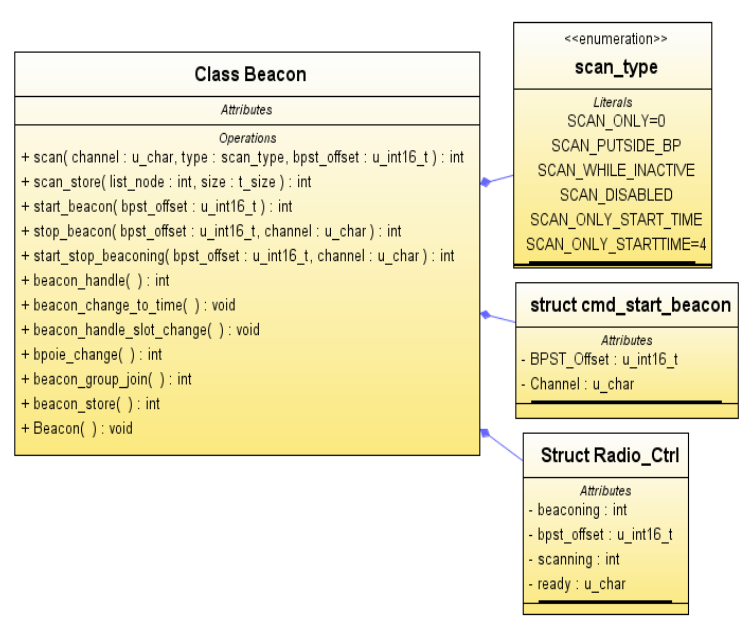

Fig. 3. Class Diagram of the Beacon Module

The beacon protocol which manages the beaconing module was implemented within the Wimedia Mac Layer to support node discovering within the same beacon group. The purpose of this module is to facilitate the process of node discovering, the temporal synchronization between more superframes, the creation and sharing of the superframe and the reduction of interference. It offers an efficient way to provide the dynamic allocation of time slots within the superframe.

Furthermore, the beacon module starts with a channel scan in order to discover all beacon time slots allowed within the Beacon Period Start Time (BPST). Then it schedules all received time slots to manage the dynamic reservation access to the superframe and allocates to each node the bandwidth it requires. The Beacon frame manages the beacon scheduling within the BPST to prevent superframe fragmentation and allowing the time slots reservation during the Data transfer Period (DTP).

Many options are added to this module to support the merging of beacons, the clustering and management of alien beacon groups. All these information were exchanged within Information Elements (IE).

The simulations concern a case study of Wireless Field Bus communication used in aerospace industry. The scenario, presented in figure 4 , is composed of central points which communicate through wireless channels with 8 nodes; each node communicates with a real time sensor at $8 \mathrm{Mb} / \mathrm{s}$ throughput rate. Depending on the need of the designed application the throughputs can vary from $65 \mathrm{Mb} / \mathrm{s}$ to $400 \mathrm{Mb} / \mathrm{s}$.

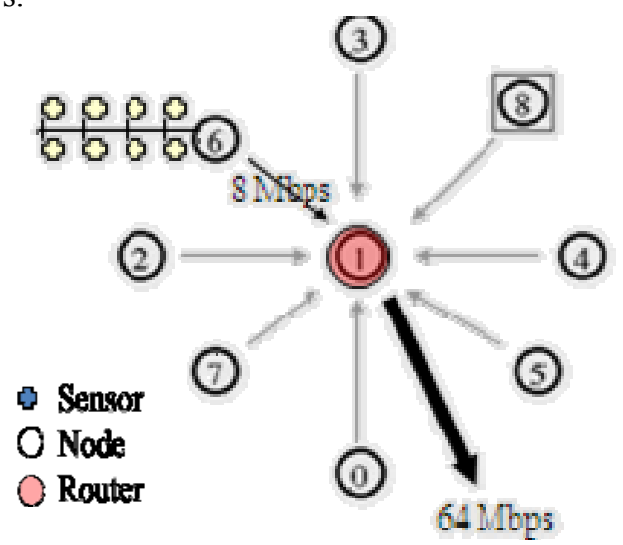

Fig. 4. Architecture of the simulated network 
The delay between two generated packets from a desired node was 2 micro-seconds. The packet size was fixed at 4095 Bytes because we used the isochronous transfer mode of the Wimedia protocol. According to the bit rate that each node can generate in the real case (8Mbits/s), we have varied this rate in order to determine the maximum rate that the router supports. The simulation results are presented in figure 5 . We conclude that the router can support the network traffic as the maximum throughput received by a Router is specified at 64 Mbits/s. In the simulator, the router can support at least $125 \mathrm{Mbits} / \mathrm{s}$.

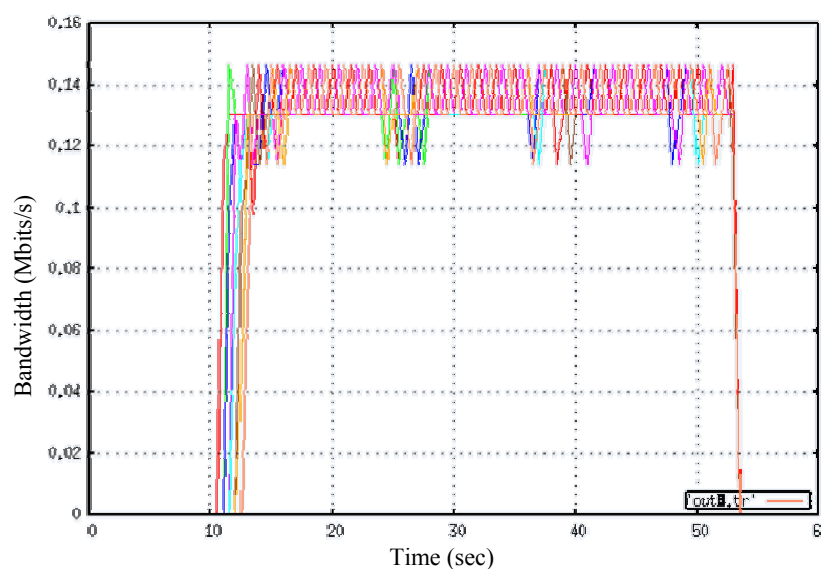

Fig. 5. Throughput at router level

In fact, the highest throughput value attempted in the simulation was at $140 \mathrm{Mbits} / \mathrm{s}$. The limit value of the simulation was attained at $125 \mathrm{Mbits} / \mathrm{s}$. We increased the packet size during the simulation and we found that the throughput increases also. The Wimedia protocol envisages a large packet size (4095 Bytes) to conserve the isochronous transfer data mode implemented within USB 2.0 and IEEE 1394. Figure 6 points out the saturation of the simulator. The simulation throughput linearly increases with the packet size up to $125 \mathrm{Mbits} / \mathrm{s}$. Then the throughput remains stable.

The linear increase of the curve proves that our protocol is more adapted for large packet sizes. The stagnation is abnormal and seems to be related to the saturation of the NS2 Simulator.

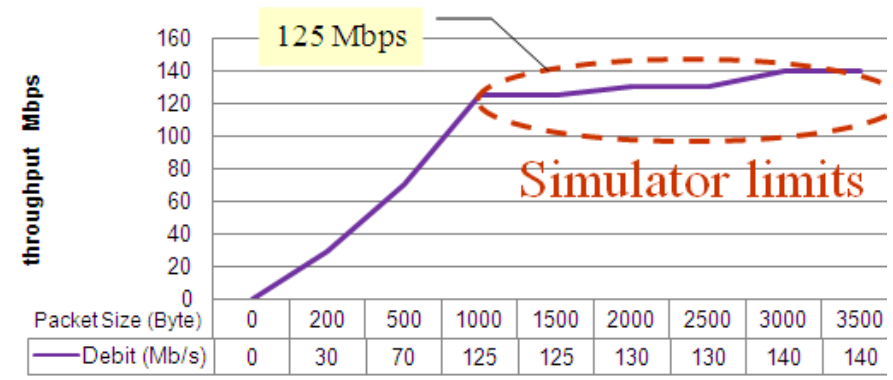

Fig. 6. NS-2 WiMedia model simulation limit

However, the simulator limit (125 Mbits/s) is higher than the requested throughput for a single router simulation (64 Mbits/s).

\section{CHANNEL EVALUATION.}

As outlined in the previous part, network simulations cannot be used alone to evaluate the possible use of the MBOFDM/Wimedia standard on a FieldBus application.

The biggest problem is that the signal that has propagated through a wireless link consists of multiple replicas of the originally transmitted signal. This phenomenon is called multipath propagation. The different multipath components are characterized by different delays and attenuations. This limits the capacity and performances of the communication system. So, analysis requires accurate channel models to determine what can be achieved and how to perform realistic measurements.

The MB-OFDM standard was evaluated essentially in strong multipath environments specified by the $802.15 .3 \mathrm{a}$ channel modeling sub-committee report (Foerster 2003). Four different measurement environments were defined, namely Channel Model (CM) 1, CM2, CM3 and CM4. CM1 describes a Line Of Sight (LOS) scenario with a separation between transmitter and receiver of less than $4 \mathrm{~m}$. CM2 describes the same range but for a Non-LOS (N-LOS) situation. CM3 describes an N-LOS scenario for distances of 4 to 10 meters between emitter and receiver. CM4 describes an environment with very strong delay dispersion, resulting in a delay spread of $25 \mathrm{~ns}$. All this data is based on measurement campaigns performed in office or industrial environment. In our application, both the propagation around a wing (application 1) and inside a satellite (application 2) cannot be modeled by these channels.

\subsection{Average White Gaussian Noise (AWGN) channel for wing propagation}

In the case of the wing propagation, the link is made between one sensor (with thin antenna) stuck on a wing and a concentrator (with horn antenna) inside the cabin. The channel is open, so there are no elements that could create a reflexion. Thus no multi path propagation takes place. The situation is summarized in the figure 7.

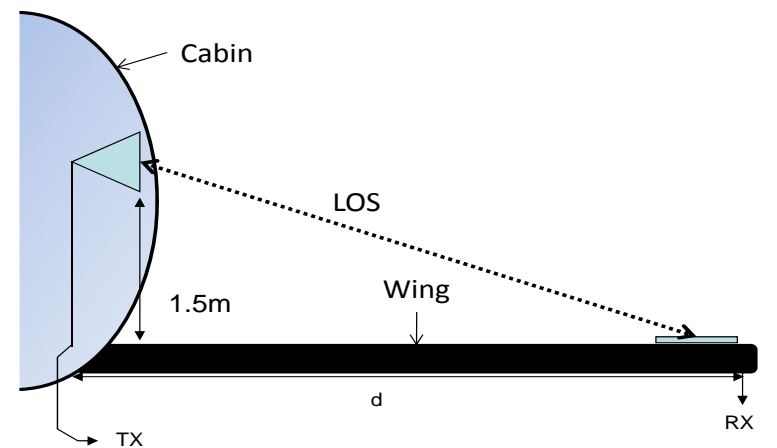

Fig. 7. "On wing” propagation conditions.

Propagation loss measurements have been done under real conditions, A340 wing, to check if the assumption of a channel without multipath is true. We compared the received power on the wing with the sent power by the emitting device. After having removed (from the measurements) the antenna gains, cable loss and impedance mismatch the 
comparison between the measured free space loss and the theoretical values are presented on figure 8 . The theoretical values correspond to the free space loss (eq.1).

$$
L_{\text {Free_Space }}=\left(\frac{\lambda}{4 . \pi . d .}\right)^{2}(1)
$$

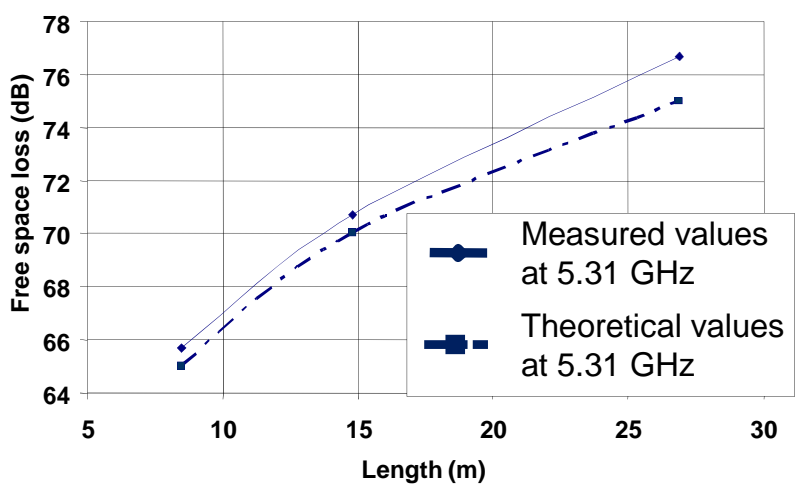

Fig. 8. Comparisons between the measured free space loss (dotted line) and the theoretical values (solid line)

The two lines have the same shape and the difference is below $1 \mathrm{~dB}$. So, we can assume that the channel in the case of an "on wing" propagation is the same as "on ground" propagation (figure 9).

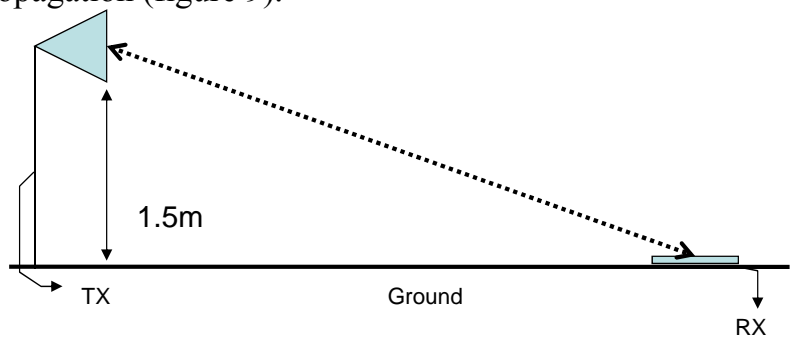

Fig. 9. Path loss measurement under "on ground" propagation conditions. The wing is replaced by ground in an open space environment.

\subsection{Metallic Closed space channel for satellite propagation}

The second application is dealing with the propagation inside a satellite. This kind of channel, where closed space propagation takes place, is presented in figure 10 and has been studied in Terral \& Pourtau (2007). A free-space behavior is obviously not suitable inside the satellite, which constitutes an electromagnetic cavity. The situation is N-LOS (like in channel model 2 or 4 ) but with metal enclosure (cavity) having large dimensions compared to the wavelength $\lambda$ of the electromagnetic field. The main assumption of the study presented in Terral \& Pourtau (2007) is that the electromagnetic environment inside the cavity is comparable to that of a reverberation chamber. This means the environment can be considered as pseudo-homogeneous and pseudo-isotropic. This point has important consequences: It implies that the orientation of antennas has no impact on the received power level. Thus the model proposed by IEEE 802.15.3a cannot be applied.

Furthermore, the two communicating elements are located in two different cavities with an aperture in the separation wall. Terral \& Pourtau (2007) shows that when the aperture becomes too large, the radiating losses through the walls increase and the uniformity of the electromagnetic environment cannot be assumed inside the cavity. This can cause an interruption of communication between the two elements depending on the size of the aperture. The particularities of this channel cannot be easily reproduced in the measurement lab. Thus, we perform error measurement tests on a mock up of the EADS-ASTRIUM satellite Eurostar 3000 .

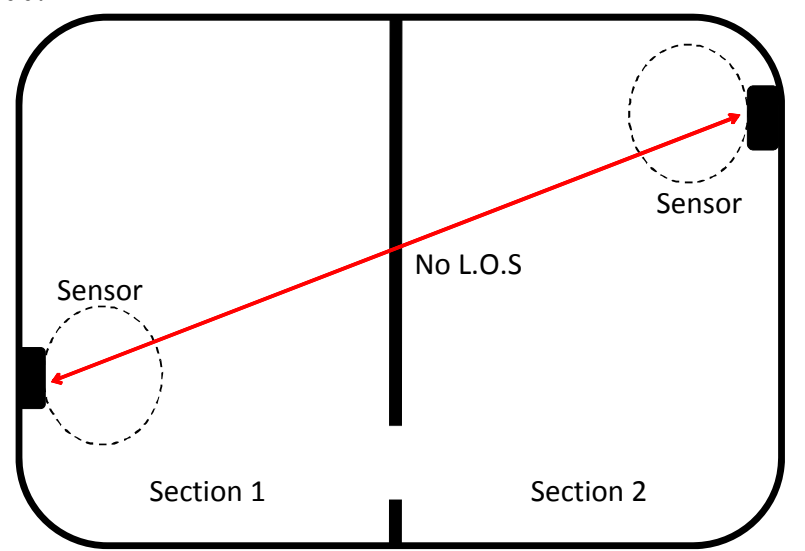

Fig. 10. "Inside satellite" propagation condition. The two elements are located in two different cavities.

\section{MEASUREMENTS PROCEDURE}

Once a modulation scheme and the channel models are well defined, we can perform tests to evaluate the error rate in our application. The MB-OFDM signal used for the experiment is generated by a commercially available evaluation kit from Wisair (Wisair 2009). The DV9110M development kit (fig 11 ) is one of the few commercially available capable of providing MB-OFDM compliant modulation with three Wimedia-MBOA sub-bands, each of $528 \mathrm{MHz}$ bandwidth in the band group 1 from $3.168 \mathrm{GHz}$ to $4.752 \mathrm{GHz}$. The average output power is UWB compliant with a value of $80 \mathrm{uW}$ ($41.3 \mathrm{dBm} / \mathrm{MHz}$ ). The hopping sequence is set as $\mathrm{fl}, \mathrm{f} 2 \mathrm{f3} \mathrm{fl}$, $\mathrm{f} 2, \mathrm{f} 3$, where $\mathrm{fl}$ is the center frequency of the lower sub-band ( $3.423 \mathrm{GHz})$, and $\mathrm{f} 2, \mathrm{f3}$ is the center frequencies of the middle $(3.960 \mathrm{GHz})$ and upper sub-bands $(4.488 \mathrm{GHz})$. The frequency spectrum of the generated signal at the output of the card is shown in figure 12.

The kit is based on the Wisair 502 SiGe-based RF Transceiver and the 531 CMOS based MAC/Baseband chips. It also includes a patented Wisair external UWB antenna, based on a PCB design and a motherboard for external Ethernet data interface (using a RJ-45 connector) and power supply control. Two laptops are used to control Wisair cards at the master side to transmit the data and the slave side to receive the data. The MAC layer of the wisair cards

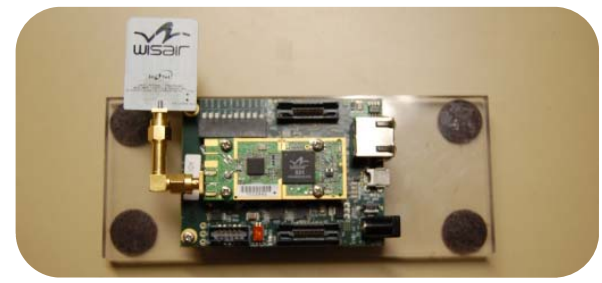

Fig. 11. Card used to perform measurements 


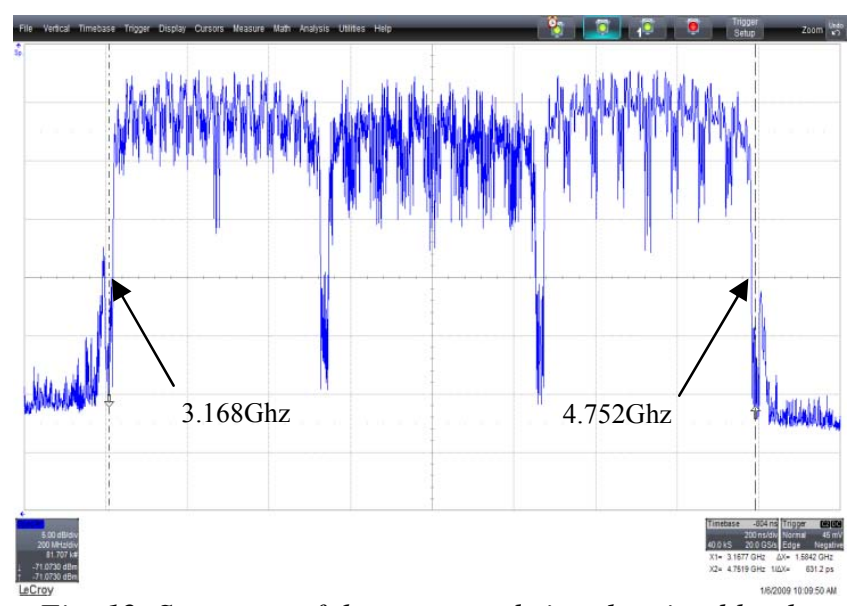

Fig. 12. Spectrum of the generated signal emitted by the card. Measured on a LECROY scope SDA 7Zi (mean on 100 periods)

Indeed, they implements the WiMedia physical layer and a Mac-Phy interface defined in the WiMedia 1.0 standard. A convenient way to use the card is through the Wisair Mini MAC layer. It does not fully implement a Wimedia MAC layer, but a simplified one. In contrary to Wimedia, the Wisair MAC layer uses the master device to synchronize the network. Figure 13 presents the MAC frames format and its encapsulation over Wimedia frames and the following section gives details on the protocol behavior.

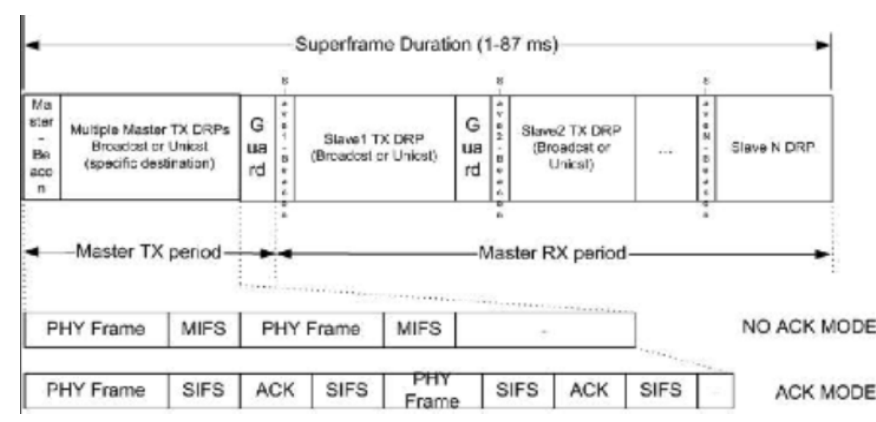

Fig. 13. MAC frames format and its encapsulation over Wimedia frames

The master device transmits a beacon as a broadcast frame. This beacon is used by the slaves for synchronization. The master then follows with data for multiple devices, where each frame is sent either as broadcast or as unicast. The MAC supports point-to-point configuration, point-to-multipoint configuration, and mesh networking. Thus the slaves can communicate either with each other or with the master. The master can communicate with all slaves. The superframe duration as well as the DRP slots (Device Reservation Periods) are configurable. The MAC supports both no-ACK and Imm-ACK policies with configurable number of retransmissions.

The configuration of the DV9110M module consists in a number of hardware registers on the device. The WisMan ${ }^{\mathrm{TM}}$ configuration and control host application gives different levels of control over these registers to configure, manage, monitor, and report status by accessing UWB devices for reading or modifying their registers. So, we can tune specific parameters, such as RF frequency and track packet traffic and error information. The quality of the link is measured along with this application. A statistics tab displays transmission and reception statistics for the device by displaying data accumulated from various device counters. We can access the number of packets sent, the number of packets retransmitted, the number of data packets dropped from transmit FIFO due to insufficient time for transmission, the number of packets correctly received or the number of packets received but discarded. We evaluated the quality of the link by comparing the number of packets sent with the number of packets correctly received. This measurement corresponds to the number of Ethernet packets that has been sent by the transmitter but never received by the receiver: the packet error rate (PER). We have performed studies with a software network analyzer (WireShark by Gerald Combs ) on the two laptops to find the number of Ethernet packets sent by laptop 1 on the Ethernet link and the number of Ethernet packets received by laptop 2 on its Ethernet port. These values correspond to the two values given by the Wisair Cards.

On each computer the Ethernet traffic is generated on UDP frames with the software Iperf (IPERF 2009). It was developed by NLANR/DAST as an alternative way to measure maximum TCP and UDP bandwidth performance. When a laptop sends a packet, the protocol encapsulation is the following: UDP over IP over Ethernet over Wisair Mac over Wimedia Phy. The packets size is set to 1480 bytes, the Maximum Transmit Unit of IP packets over Ethernet.

\section{ERROR RATE RESULTS}

We performed Frame Error Rate measurements in three kinds of environment: Office Like (closed room with many electronic devices), inside a Satellite (see section 4.1) and "on ground" (see section 4.2).

\subsection{Frame Error rate measurement indoor}

The experiment is conducted in a $5 \times 5$ room (figure 14). The Packet Error Rate (PER) was measured on a network of two UWB elements (piloted by two laptops) for a distance between the emitter and the receiver of 1, 2, 2.5 and 3 meters. All the results are presented in table 1.

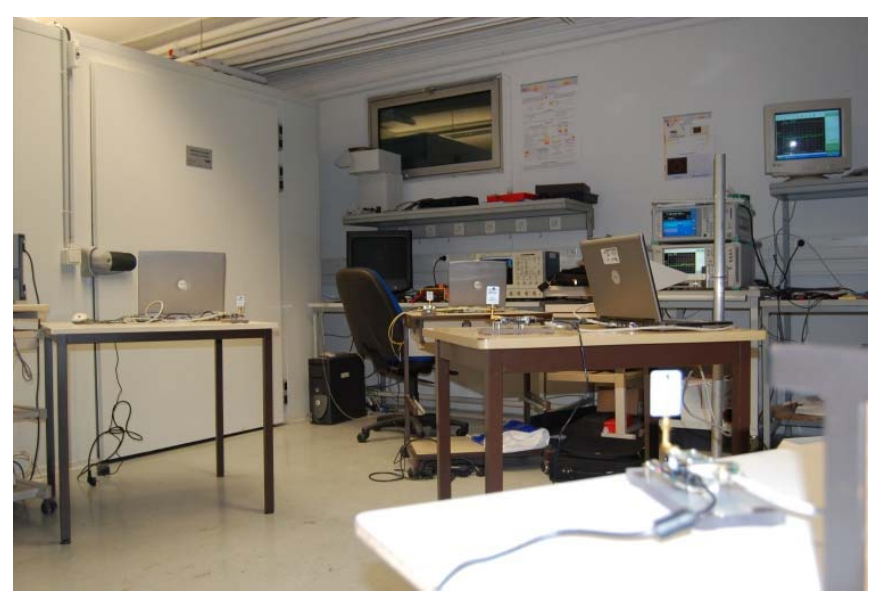

Fig. 14. Lab room used where measures have been performed 
Table. 1. Packet Error Rates in the lab room.

\begin{tabular}{|c|c|c|c|c|}
\hline Data rate & $\begin{array}{c}\text { Distance } \\
\text { (m) }\end{array}$ & $\begin{array}{c}\mathrm{Nb} \text { of } \\
\text { measures }\end{array}$ & $\begin{array}{c}\text { Packet } \\
\text { Error } \\
\text { rate } \\
\text { (mean) }\end{array}$ & $\begin{array}{l}\text { Standard } \\
\text { deviation }\end{array}$ \\
\hline \multirow{4}{*}{$\begin{array}{c}53.3-320 \\
\text { Mbits/s }\end{array}$} & 1 & 10 & $<0.1 \%$ & 0.0 \\
\hline & 2 & 10 & $<0.1 \%$ & 0.0 \\
\hline & 2.5 & 10 & $<0.1 \%$ & 0.0 \\
\hline & 3 & 10 & $<0.1 \%$ & 0.0 \\
\hline \multirow{3}{*}{$\begin{array}{c}400 \\
\text { Mbits/s }\end{array}$} & 1 & 11 & $<0.1 \%$ & 0.0 \\
\hline & 2 & 6 & $<0.1 \%$ & 0.0 \\
\hline & 3 & 12 & $1 \%$ & 0.1 \\
\hline \multirow{4}{*}{$\begin{array}{c}480 \\
\text { Mbits/s }\end{array}$} & 1 & 15 & $1 \%$ & 0.1 \\
\hline & 2 & 10 & $2 \%$ & 0.1 \\
\hline & 2.5 & 9 & $1 \%$ & 0.0 \\
\hline & 3 & 17 & $82 \%$ & 0.1 \\
\hline
\end{tabular}

The measurement environment corresponds to the channel model 1 (LOS and less than $4 \mathrm{~m}$ between the communicating elements) of IEEE 802.15.3a where the ranges necessary to achieve a PER of $8 \%$ are presented in table 2 . The $8 \%$ value for the PER is equivalent to a Bit Error Rate (BER) of 1e-5 for a 1024 byte frame body. In our measurements, the link is excellent (less than 1\% of PER) for data rates below 400 Mbits/s between 1 and 3 meters. For $480 \mathrm{Mbits} / \mathrm{s}$ the link is perfect for distance below $2.5 \mathrm{~m}$. So, by these measurements we have confirmed the performance announced by the standard (Batra 2003) for the standardized channel models.

Table. 2. Simulated range to achieve a PER of $8 \%$ with a 90\% link success probability (Batra 2003).

\begin{tabular}{|c|c|c|c|}
\hline Data Rate & AWGN & CM1 & CM2 \\
\hline $110 \mathrm{Mbits} / \mathrm{s}$ & $20.5 \mathrm{~m}$ & $11.4 \mathrm{~m}$ & $10.7 \mathrm{~m}$ \\
\hline $200 \mathrm{Mbits} / \mathrm{s}$ & $14.1 \mathrm{~m}$ & $6.9 \mathrm{~m}$ & $6.3 \mathrm{~m}$ \\
\hline $480 \mathrm{Mbits} / \mathrm{s}$ & $7.8 \mathrm{~m}$ & $2.9 \mathrm{~m}$ & $6.6 \mathrm{~m}$ \\
\hline
\end{tabular}

\subsection{Frame Error rate measurement inside a satellite}

The second measurement campaign has been performed on a Eurostar 3000 mock-up. To validate the propagation, the emitter and receiver are first placed in the same compartment of the satellite (LOS with a distance of $0.75 \mathrm{~m}$ ). In this situation, the packet error rate is extremely low for all data rates except for 400 and $480 \mathrm{Mbits} / \mathrm{s}$ with measured values between $0.1 \%$ and $1.10 \%$ (mean on 5 measurements). For the two highest data rates, the measured PER is $61.7 \%$ and $100 \%$, respectively. The environment is so poor, due to the small size of the cavity and the metallic walls, that the error correcting codes and redundancies for these data rates are not enough to fight correctly against multipath and attenuation. So, in the other tests, we limit the data rate below $400 \mathrm{Mbit} / \mathrm{s}$. The two cards have then been put in different compartments producing a scenario like the one presented on figure 15 . The two cards are separated by a metallic wall. So, there is no Line of Sight. There is a hole between the two cavities and the outside of the satellite. The size of the hole is around $30 \mathrm{~cm} \times 30 \mathrm{~cm}$, it is located 1 meter above the two cards. The hole connects the two cavities and the outside of the satellite as shown in figure 15 .

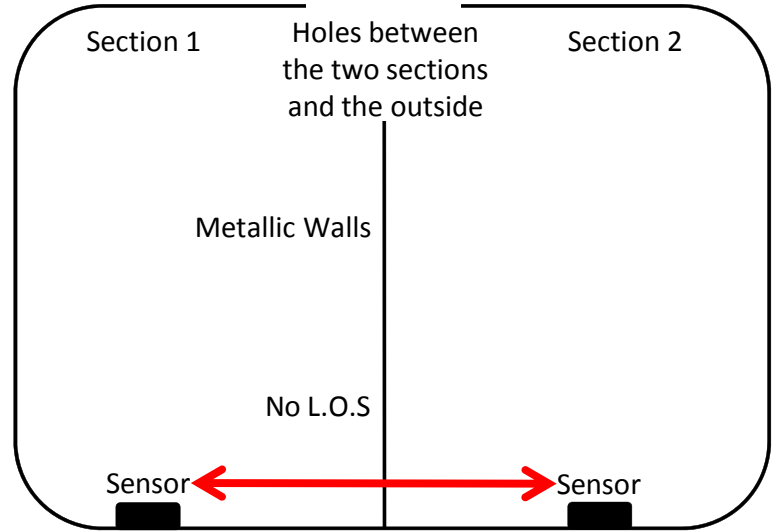

Fig. 15. “Inside satellite” packet error rate measurements.

In this scenario, the PER is always above $10 \%$. All the results are shown in the Table 3. Even data rates below $100 \mathrm{Mbits} / \mathrm{s}$ are not to achieve. Indeed, the quality (power and phase) of information received is so weak, due to metallic walls, that even beacons sent by the master cannot be interpreted by the slave. The presence of a hole between the two cavities and the outside can make things even worse. Thus we decided to close this opening with by a metallic element. Thereupon, the PER decreases but the gain is only 5 to 10 percentage points.

Table. 3. Packet Error Rates inside the satellite

\begin{tabular}{|c|c|c|c|c|}
\hline $\begin{array}{c}\text { Data } \\
\text { rate } \\
(\text { Mbits/s })\end{array}$ & $\begin{array}{c}\text { Nb of } \\
\text { measures }\end{array}$ & $\begin{array}{c}\text { Frame } \\
\text { Error rate } \\
\text { with a hole } \\
\text { between the } \\
2 \text { cavities }\end{array}$ & $\begin{array}{c}\text { Frame } \\
\text { Error rate } \\
\text { with the } \\
\text { hole closed } \\
\text { by metallic } \\
\text { elements }\end{array}$ & $\begin{array}{c}\text { Gain } \\
\text { of the } \\
\text { closed } \\
\text { hole }\end{array}$ \\
\hline 53.3 & 5 & $86.2 \%$ & $71.9 \%$ & 14 \\
\hline 80 & 5 & $87.4 \%$ & $73.1 \%$ & 14 \\
\hline 106 & 3 & $88.2 \%$ & $77.3 \%$ & 11 \\
\hline 160 & 4 & $100 \%$ & $100 \%$ & NA \\
\hline 200 & 3 & $100 \%$ & $100 \%$ & NA \\
\hline 320 & 3 & $100 \%$ & $100 \%$ & NA \\
\hline
\end{tabular}

In such a configuration, the MB-OFDM as implemented in the Wisair kit cannot be used. So, two different directions of research are followed. The goal of the first one is to develop passive devices that could be placed in the hole between the cavities to redirect the signal from one closed space to the other (Ali et al. 2009). The goal of the second is to adapt the physical layer to this kind of very specific environment (closed space with metallic wall and N-LOS) without modifying the achievable data rate.

\subsection{Frame Error rate measurement outdoor - "on ground" propagation}

The last measurement campaign has been done outside in an open space situation in order to recreate the "on wing" channel (figure 16). The emitter and receiver are placed one meter above the ground (figure 17). 


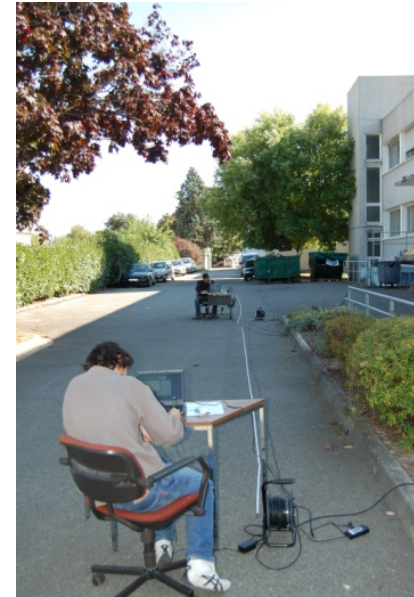

Fig. 16. Environment used for the "on ground" packet error rate measurements.

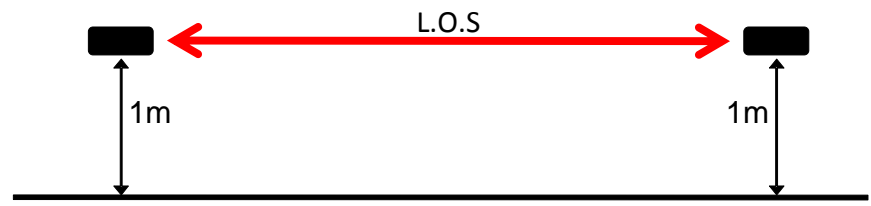

Ground

Fig. 17. Schematic representation of the emitter and receiver position

The PER is evaluated for each data rate at a distance form $1 \mathrm{~m}$ to $15 \mathrm{~m}$ (maximum length on an aircraft wing). The results are presented in table $4 \mathrm{a}$ and $4 \mathrm{~b}$ and figure 18 .

Table. 4a. Packet Error Rates for outdoor "on ground" propagation from $1 \mathrm{~m}$ to $5 \mathrm{~m}$

\begin{tabular}{|c|c|c|c|c|}
\hline \multirow{2}{*}{$\begin{array}{c}\text { Data rate } \\
(\text { Mbits/s) }\end{array}$} & \multicolumn{4}{|c|}{$\begin{array}{c}\text { Packet Error Rate } \\
\text { for each distance }\end{array}$} \\
\cline { 2 - 5 } & $1 \mathrm{~m}$ & $2 \mathrm{~m}$ & $3 \mathrm{~m}$ & $5 \mathrm{~m}$ \\
\hline 53.3 & $<0.01 \%$ & $<0.01 \%$ & $<0.01 \%$ & $<0.01 \%$ \\
\hline 80 & $<0.01 \%$ & $<0.01 \%$ & $<0.01 \%$ & $<0.01 \%$ \\
\hline 106.7 & $<0.01 \%$ & $<0.01 \%$ & $<0.01 \%$ & $<0.01 \%$ \\
\hline 160 & $<0.01 \%$ & $<0.01 \%$ & $<0.01 \%$ & $<0.01 \%$ \\
\hline 200 & $<0.01 \%$ & $<0.01 \%$ & $<0.01 \%$ & $<0.01 \%$ \\
\hline 320 & $<0.01 \%$ & $<0.01 \%$ & $<0.01 \%$ & $<0.01 \%$ \\
\hline 400 & $0.01 \%$ & $0.04 \%$ & $0.2 \%$ & $0.6 \%$ \\
\hline
\end{tabular}

Table. 4a. Packet Error Rates for outdoor “on ground” propagation from $7 \mathrm{~m}$ to $15 \mathrm{~m}$

\begin{tabular}{|c|c|c|c|c|}
\hline \multirow{2}{*}{$\begin{array}{c}\text { Data rate } \\
\text { (Mbits/s) }\end{array}$} & \multicolumn{4}{|c|}{$\begin{array}{c}\text { Packet Error Rate } \\
\text { for each distance }\end{array}$} \\
\cline { 2 - 5 } & $7 \mathrm{~m}$ & $10 \mathrm{~m}$ & $12 \mathrm{~m}$ & $15 \mathrm{~m}$ \\
\hline 53.3 & $<0.01 \%$ & $6.2 \%$ & $0.3 \%$ & $2.23 \%$ \\
\hline 80 & $<0.01 \%$ & $11.9 \%$ & $0.5 \%$ & $5.51 \%$ \\
\hline 106.7 & $<0.01 \%$ & $16.1 \%$ & $0.7 \%$ & $8.5 \%$ \\
\hline 160 & $<0.01 \%$ & $18.8 \%$ & $1.2 \%$ & $100 \%$ \\
\hline 200 & $0.5 \%$ & $18.3 \%$ & $2 \%$ & $100 \%$ \\
\hline 320 & $18.6 \%$ & $25.6 \%$ & $8 \%$ & $100 \%$ \\
\hline 400 & $100 \%$ & $100 \%$ & $95 \%$ & $100 \%$ \\
\hline
\end{tabular}

The error rates are low and sufficient to establish a good communication for distances from 1 to $12 \mathrm{~m}$, with an exception at $10 \mathrm{~m}$. More precisely, table $4 \mathrm{a}$ shows that the maximum data rate is achievable for distances below 5 meters. But the PER is below $8 \%$ only for data rates below $200 \mathrm{Mbits} / \mathrm{s}$ (table 4b) when the distance is between $7 \mathrm{~m}$ to $12 \mathrm{~m}$. The propagation channel is very close to an AWGN channel. So the results should resemble the simulations extracted from Batra 2003 (table 2). The comparison between these results and the measured ones are presented in table 5.

Table. 5. Comparison between simulated and measured range below which a PER of $8 \%$ is achieved

\begin{tabular}{|c|c|c|}
\hline Data Rate & Batra 2003 & Measurements \\
\hline 110 Mbits $/ \mathrm{s}$ & $20.5 \mathrm{~m}$ & $>15 \mathrm{~m}$ \\
\hline $200 \mathrm{Mbits} / \mathrm{s}$ & $14.1 \mathrm{~m}$ & $12-13 \mathrm{~m}$ \\
\hline $480 \mathrm{Mbits} / \mathrm{s}$ & $7.8 \mathrm{~m}$ & $6-7 \mathrm{~m}(400 \mathrm{Mbits} / \mathrm{s})$ \\
\hline
\end{tabular}

These results correspond quite well. However, in table $4 \mathrm{~b}$ there is a strong increase of the error rate at $10 \mathrm{~m}$. A first explanation of this phenomenon could be the fact that at this particular point there is a destructive interference due to multipath effects. Indeed, the antennas are located at one meter height (above the ground) so there are (few) multiple paths due to reflexions on the ground. These multipaths could induce destructive interference and explain the increase of loss.

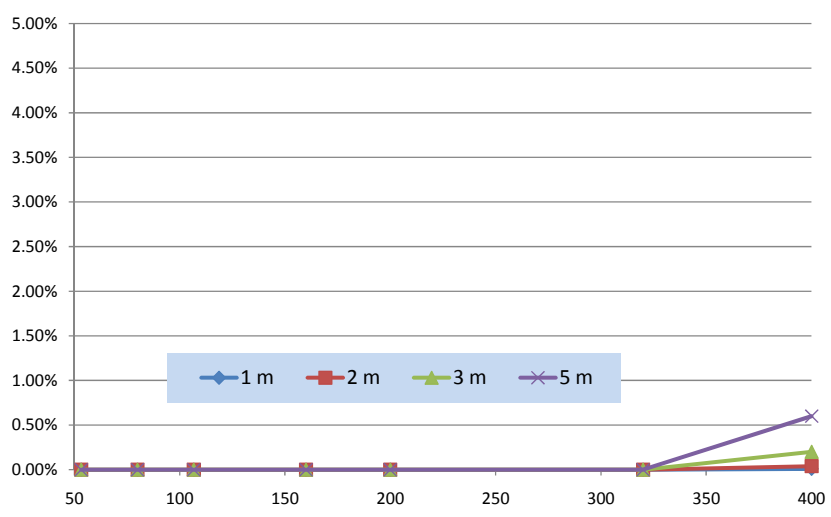

Fig. 18a. Packet Error Rates for outdoor "on ground” propagation from $1 \mathrm{~m}$ to $5 \mathrm{~m}$

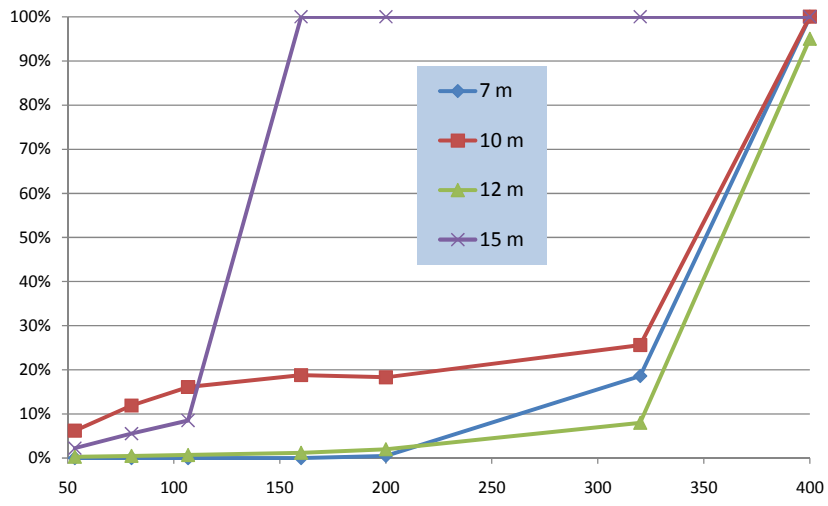

Fig. 18b. Packet Error Rates for outdoor "on ground” propagation from $5 \mathrm{~m}$ to $15 \mathrm{~m}$ 
Measurements with refined distance coverage (one measurement every $0.5 \mathrm{~m}$ from $5 \mathrm{~m}$ to $10 \mathrm{~m}$ ) at the specific data rate of $200 \mathrm{Mbits} / \mathrm{s}$ yield the results presented in figure 19. We observe that there are two zones of destructive interferences at $6 \mathrm{~m}$ and at $9.5 \mathrm{~m}$. With a very simple model of propagation (two ray ground reflection model) we can almost confirm these particular values.

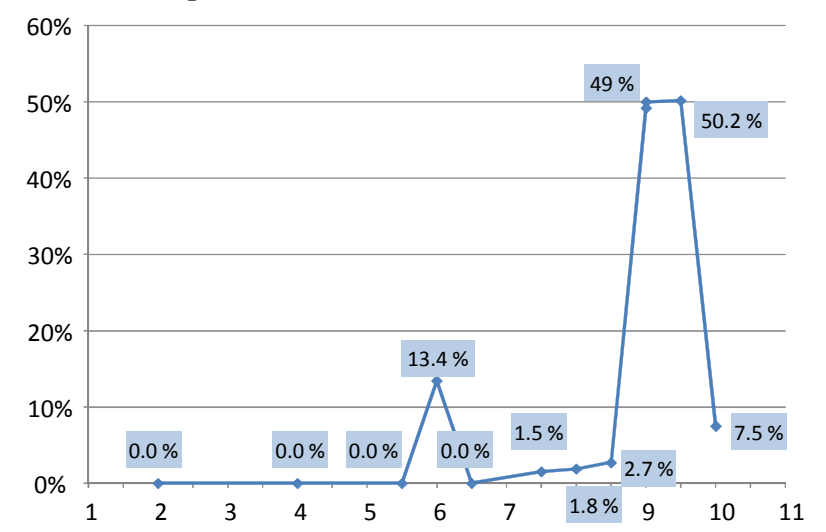

Fig. 19. Packet Error Rates for 200 Mbits/s, refined distance resolution

The MB-OFDM modulation scheme is very robust against multipath in indoor environments because it is built to retrieve the energy from all the multipaths. There is always a transmission path between the emitter to the receiver to bring the information. But when there is very few multipath, destructive interference appears and deteriorate the predicted PER.

In spite of the many measurements done, we notice another surprising behaviour: The measurements seem to be very dependant of the campaigns. The results presented are the most common ones, but at some campaigns the error rate peaks are located at very different values (different from $6 \mathrm{~m}$ and $9.5 \mathrm{~m}$ ) or error rates are increasing dramatically even at low distance. In such situations, the quality of the communication is strongly dependent of weather and atmospheric conditions.

The standard was developed for indoor propagation but has some strange behaviour in outdoor condition.

\section{CONCLUSION}

In this paper we present results from experiments which aim to evaluate the possibility of using the MB-OFDM modulation scheme as implemented in commercially available kits in a wireless field bus for aerospace industry hardware system testing. This powerful modulation is new in the field of Wireless Sensor networks but has decisive advantages for high data rate applications. Simulations have been performed on a software network simulator but do not offer enough results to validate this modulation scheme.

Frame error rate measurements have been performed indoor, inside a satellite and outdoor. We observe that the MBOFDM standard works very well in indoor environment (compliant to the standard) but the behaviour inside a satellite and outdoor is not satisfying and both the precise reasons of this behaviour and possible solutions have to be investigated further.

\section{REFERENCES}

Ali, A., Coustou, A. \& Aubert, H., 2009. Wide Band Passive Repeaters: Design and Measurements. In Proceedings of the IEEE International Symposium on Antennas \& Propagation. Charleston, SC (USA), p. To be published.

Batra, A., 2003. Multi-band OFDM Physical Layer Proposal for IEEE 802.15 Task Group 3a, IEEE P802.15 Working Group for Wireless Personal Area Networks (WPANs). Available at: http:/grouper.ieee.org/groups/802/15/pub/2003/Jul0 3/03268r2P802-15_TG3a-Multi-band-CFPDocument.pdf [Accessed March 22, 2009].

Batra, A., Balakrishnan, J. \& Dabak, A., 2004. Multi-band OFDM: a new approach for UWB. In Circuits and Systems, 2004. ISCAS '04. Proceedings of the 2004 International Symposium on. pp. 365-368 Vol.5.

ECMA, 2008. High Rate Ultra Wideband PHY and MAC Standard, ECMA international. Available at: http://www.ecma-

international.org/publications/files/ECMAST/ECMA-368.pdf [Accessed March 21, 2009].

Foerster, E., 2003. Channel modeling sub-committee report final, Document IEEE P802.15-02/490r1-SG3a. Available

at: http://www.ieee802.org/15/pub/2003/Mar03/02490r 1P802-15_SG3a-Channel-Modeling-SubcommitteeReport-Final.zip [Accessed August 3, 2009].

Henaut, J., Dragomirescu, D. \& Plana, R., 2009. FPGA Based High Data Rate Radio Interfaces for Aerospace Wireless Sensor Systems. In Proceedings of the fourth International Conference On Sytems. Cancun (Mexico): IARIA, pp. 173-178.

Henaut, J. et al., 2008. Radio Interface for High Data Rate Wireless Sensor Networks. In Proceedings of the International Multi-Conference on Engineering and Technological innovation. Orlando, Fl (USA): IIIS, pp. 104-109.

IPERF, 2009. iperf. Available at: http://sourceforge.net/projects/iperf/ [Accessed March 21, 2009].

Lecointre, A., Dragomirescu, D. \& Plana, R., 2008. Design and Hardware Implementation of a Reconfigurable Mostly Digital IR-UWB Radio. Romanian Journal of Science and Technology, vol 11(nr 4), 295-318.

Terral, M. \& Pourtau, J., 2007. Reducing EMC verifications for future telecommunication Platforms. Reducing EMC verifications for future telecommunication Platforms, Alcatel Alenia Space.

Wisair, 2009. Commercial Website. Available at: http://www.wisair.com/ [Accessed March 23, 2009]. 